\title{
Las consecuencias del urbanismo fragmentador en la vida cotidiana de habitantes de la ciudad de Santiago de Chile
}

Paola Jirón. Universidad de Chile, Santiago, Chile.

Pablo Mansilla. Universidad de Chile, Santiago, Chile.

RESUMEN | En el contexto de las múltiples transformaciones urbanas que experimentan las grandes metrópolis, este trabajo analiza el modo en que, por medio de diversas intervenciones, la práctica del urbanismo fragmenta las condiciones espacio-temporales sobre las cuales se constituye la espacialidad urbana de la vida cotidiana. Con un enfoque etnográfico de la movilidad cotidiana de los habitantes de la ciudad de Santiago, describe el modo en que la fragmentación urbana es vivida por los habitantes, los que implementan diversas estrategias de movilidad para zurcir las espacialidades inconexas, interrumpidas y segregadas en las que ha sido descompuesta la ciudad, y que han ido fragmentando la espacialidad de la vida cotidiana. Los resultados de la investigación dan cuenta de las consecuencias de este urbanismo fragmentador, en particular en las condiciones de exclusión social, y también permiten proponer posibles intervenciones urbanas destinadas a aminorar el impacto de dicha fragmentación y consiguiente exclusión.

PALABRAS CLAVE | fragmentación urbana, movilidad, urbanismo.

ABSTRACT | In the context of the multiple urban transformations taking place in large metropoles, this paper analyzes, through various means, the way in which contemporary ur $\rightarrow$ banism practices serve to fragment the spatial-temporal conditions upon which the spatiality of daily urban life is constituted. An ethnographic approach to the everyday mobility of Santiago's residents is applied, in order to describe the way in which urban dwellers experience urban fragmentation by devising different mobility strategies to weave their way through the disconnected, interrupted and segregated spatialities into which the city has been decomposed, resulting in fragmentation of the spatial aspects of daily life. The research results account for the consequences of this 'fragmenting urbanism', demonstrating effects regarding the conditions of social exclusion. The results are considered to be useful for the proposal of possible urban interventions designed to lower the impact of fragmentation and the resulting exclusion.

KEY WORDS | fragmentation, mobility, urbanism.

Recibido el 26 de diciembre de 2012, aprobado el 6 de abril de 2013

E-mail: Paola Jirón, paolajiron@uchilefau.cl I Pablo Mansilla, pablo.manq@gmail.com 


\section{Introducción}

En la fase actual de la globalización capitalista es necesario identificar dos aspectos de considerable incidencia en las transformaciones experimentadas por las ciudades comprendidas en ese orbe. El primero se vincula con las reformas político-económicas que han redefinido los objetivos del desarrollo de esos países durante las últimas décadas, y que en casos como el de Chile han implicado la adopción del modelo neoliberal, potenciando procesos de reestructuración urbana de graves consecuencias sobre la ciudad actual (De Mattos, 2002b; 2010). Un segundo aspecto tiene relación con las innovaciones tecnológicas en los sistemas de transportes y telecomunicaciones, que han permitido comprimir los tiempos de desplazamientos de carácter financiero, informacional y humano, creando un canal adecuado para la hipermovilidad del capital y su intervención en la ciudad (Santos, 1994; Harvey, 1994; 2004). Ambos tipos de cambios -político-económicos y tecnológico-comunicacionales- están en la raíz de parte de las transformaciones estructurales que las condiciones espacio-temporales de las ciudades enfrentan en la actualidad.

En el contexto de estas transformaciones, la fragmentación espacial es uno de los fenómenos urbanos que se imprimen con más fuerza sobre la ciudad contemporánea. Como lo mencionan Caprón y González (2006), por medio del concepto de fragmentación se busca dar cuenta de una ruptura en la concepción integral de la ciudad. Este concepto, propio de la física, ha sido extrapolado a los estudios urbanos para dar cuenta de "una unidad homogénea, autónoma, donde importa poco la diferenciación con los otros fragmentos, replicándose en el escalón siguiente hasta el infinito" (Ibíd., p. 65). Con él se hace referencia a la segmentación y la atomización del espacio urbano, las que generan discontinuidades espaciales que limitan la unificación del conjunto urbano. La noción de fragmentación permite, entonces, describir las lógicas de separación, extensión y nuevas fronteras urbanas, que establecen distinciones entre los diversos grupos sociales.

Los enfoques a partir de los cuales se indaga en estas transformaciones han sido clarificadores respecto del modo en que la fragmentación se materializa en la morfología urbana, esto es, en la estructura y funcionamiento de la ciudad como escenario de desigualdades (véase al respecto Borsdorf, 2003; Janoschka, 2002, 2006; Hidalgo, 2004; Hidalgo, Borsdorf \& Sánchez, 2007; Prévôt-Schapira \& Cattaneo, 2008; Cariola \& Lacabanca, 2001; Escolano, 2007; Link, 2008; Ortiz \& Améstica, 2011; Rodríguez \& Winchester, 2005; Parnreiter, 2003). No obstante, la discusión en torno al tema posee algunas debilidades que es necesario revisar.

Una primera debilidad se encuentra vinculada a una discusión respecto de la fragmentación del tiempo que aporte a una comprensión espacio-temporal de este fenómeno. Tal discusión ha sido prácticamente nula. La ciudad aparece en las investigaciones urbanas como un hecho atemporal. En esta perspectiva se genera una disociación entre tiempo y espacio y, como consecuencia, que los conceptos y categorías de análisis a los que se refieren sus autores para describir las ciudades sean fijos y estáticos (Jirón, Lange y Bertrand, 2010; Lange, 2011; Hiernaux, 2006; Salvador, 2000). Tampoco han sido incorporados en esta discusión otros conceptos asociados 
al tiempo, como los enfoques de movilidad, de ritmos o de accesibilidad en la vida cotidiana (Lefebvre, 2004; Jirón, 2010a; Lange, 2011).

Una segunda debilidad en los estudios de fragmentación urbana ha sido destacada por Correa (1989), Caprón y González (2006) y Valdés (2007), cuyas críticas a los estudios de fragmentación se basan en que estos no contemplan las interacciones que ocurren entre los fragmentos urbanos. Los estudios urbanos han incurrido en una serie de sesgos al concentrarse únicamente en el análisis de lo que sucede al interior de un fragmento urbano -por ejemplo, analizando un barrio cerrado de la ciudad-, sin comprender las interacciones que este espacio mantiene con otros fragmentos espaciales de la ciudad, donde se concentran, por ejemplo, el trabajo, la educación u otras actividades. Con el fin de profundizar sobre esta perspectiva es necesario explorar el concepto de fragmentación integrando una discusión epistemológica respecto de nociones asociadas a la movilidad, los flujos, la interacción y las trayectorias. Del mismo modo, puede ser interesante tensionar este tipo de análisis ampliándolo a un cuestionamiento de la escala en que se realizan las observaciones de la fragmentación (Caprón \& González, 2006; Mansilla, 2011a; Smith, 2001; Brenner, 2001), para situar el fragmento en el contexto de una totalidad mayor que la del recorte espacial que se está proyectando.

En tercer lugar, existe debilidad al dar cuenta de las consecuencias que el urbanismo contemporáneo genera en la vida cotidiana de las personas que habitan la ciudad. Los estudios urbanos han centrado su interés en el diseño, la forma, la estructura, la materialidad y la morfología urbanas como contenedores de las ideas y las acciones de los grupos sociales. Sin embargo, rara vez se integra en el análisis al sujeto y su experiencia diaria o se indaga en cómo se configura la espacialidad de la vida cotidiana. Con el fin de lograr una visión totalizante y generalizadora que permita construir modelos, teorías y leyes generales respecto de la ciudad, se elimina de los estudios urbanos la diversidad de experiencias que acontecen en la vida cotidiana de sus habitantes, ya que, para la mirada urbanística, la heterogeneidad de experiencias que se constituyen a partir de las significaciones personales del mundo limita la posibilidad de encontrar un punto de partida para la intervención de la realidad.

A partir de estas tres debilidades en la discusión respecto de la fragmentación urbana hoy, el presente artículo intenta analizar el urbanismo como fragmentador de las condiciones espacio-temporal en la vida cotidiana. Más que un estado, se argumenta que el urbanismo fragmentador es un proceso, una serie de acciones, prácticas e intervenciones sistemáticas sobre las condiciones físico-espaciales de la ciudad, pero sobre todo en la relación de las personas con el espacio urbano, todas ellas operaciones que generan un estado de fragmentación.

El presente artículo intenta responder estos cuestionamientos por medio de las herramientas teórico-metodológicas del debate sobre movilidad en las ciencias sociales (Elliot \& Urry, 2010; Sheller \& Urry, 2006; Cresswell, 2006, Kaufman, 2002; Ascher, 2006). Las prácticas de movilidad en que las personas se desenvuelven diariamente para acceder a actividades, relaciones o lugares (Jirón \& Mansilla, 2013) permiten visualizar el modo en que los límites de los fragmentos espaciales en los cuales la ciudad ha sido descompuesta son transgredidos por medio de la movilidad, 
reunificándose sus partes por medio de estrategias que permiten subvertir el orden impuesto por el urbanismo actual.

Para lograr el propósito señalado, primero se presenta la discusión actual sobre fragmentación urbana. Luego se discuten las carencias que se detectan en el análisis de tres ámbitos: la fragmentación temporal, la movilidad y el urbanismo fragmentador. Posteriormente, con el fin de comprender las consecuencias de este urbanismo, este trabajo explora la fragmentación de la vida de las personas y su cotidianidad, utilizando para ello etnografías de movilidad cotidiana de viajeros urbanos. El enfoque metodológico se aplica a los viajes desde el lugar de residencia a los múltiples destinos que forman parte de la vida diaria de las personas. Este enfoque facilita la comprensión del modo en que los habitantes urbanos, de manera independiente y privada, deben resolver cómo unir sus vidas en la ciudad, utilizando la movilidad para zurcir las partes de esta realidad fragmentada. Es en este movimiento donde se manifiestan algunas de las consecuencias de la actual forma de hacer ciudad.

\section{Ciudades fragmentadas y fragmentación urbana}

En términos amplios, la bibliografía de los últimos diez años respecto de ciudades latinoamericanas es clara en evidenciar la fragmentación como una de las principales características de las grandes metrópolis (véase Prévôt-Schapira, 2001; Cariola \& Lacabanca, 2001; Janoshka, 2002; De Mattos, 2002a; Borsdoff, 2003; Parnreiter, 2003; Rodríguez \& Winchester, 2005; Hidalgo, 2004; Janoshka, 2006; Escolano, 2007; Hidalgo et al., 2007; Prévôt-Schapira \& Cattaneo, 2008; Link, 2008; VidalKoppman, 2009; Ortiz \& Améstica, 2011; Fani, 2011).

Las investigaciones citadas evidencian dos líneas de análisis respecto del proceso de fragmentación urbana: la primera relacionada con las discontinuidades en el proceso de expansión urbana respecto de su trama; y la segunda, ligada a procesos de desigualdad social y barreras materiales o inmateriales de esta fragmentación (Valdés, 2007).

Para la primera, los estudios orientados a la explicación de las discontinuidades en el proceso de fragmentación urbana se refieren, según De Mattos (2002a), al actual proceso de revalorización del papel de las áreas metropolitanas en el funcionamiento de la economía capitalista, el que comporta una serie de transformaciones urbanas que cambian sustancialmente la estructura y funcionamiento de la ciudad (Link, 2008). Tal tendencia genera una súper especialización funcional e interconectada en la ciudad, que se traduce en múltiples centros especializados, de comercio, industria, servicios o residencias, que obedecen a procesos diferentes entre sí, pero que se relacionan en la configuración de una trama de funciones especiales, complementarias, interdependientes, conectadas y marcadamente discriminantes (Link, 2008). Este proceso genera un paisaje dual entre sectores exitosos de la ciudad global y sectores marginados de los beneficios de la modernización (De Mattos, 2006); es decir, la ciudad se fragmenta en espacios ganadores y perdedores, territorios iluminados y oscuros, islas de riqueza sobre mares de precariedad y vulnerabilidad (Caravaca, 1998; Veltz, 1999). 
Borsdorf (2007) denomina este proceso como "fragmentación", una nueva forma de separación de funciones y elementos socioespaciales, ya no -como antesreferida a la totalidad de la ciudad (ciudad rica-ciudad pobre, zona habitacionalzona industrial), sino en una dimensión menor: la fragmentación urbana como la disposición de núcleos de riqueza incrustados en territorios de pobreza. Tal situación se encuentra reforzada por la construcción de muros y otros dispositivos de seguridad, que separan y aseguran el distanciamiento entre la pobreza y los enclaves de riqueza. Asimismo, la construcción de autopistas urbanas permite el desarrollo de esta nueva organización espacial, al unir los fragmentos distantes en los que ha sido descompuesta la ciudad. Esta ya no se constituye a partir de zonas bien diferenciadas de los grupos sociales, sino que ve su espacio fragmentado y segregado en pequeños barrios homogéneos (Borsdorf, Bähr \& Janoschka, 2002). En este sentido, es importante destacar la distinción que Caprón y González (2006) hacen entre los procesos de segregación y los de fragmentación. La segregación, según los autores, da cuenta de una ciudad que se encuentra formada por partes -no por fragmentos- que mantienen relaciones de complementariedad, pero también de oposición. La fragmentación, en tanto, da cuenta de fraccionamientos cerrados con relativa autonomía y aislamiento espacial, aunque los múltiples impactos generados a partir de estos fraccionamientos influyen en diversos espacios del contexto urbano.

La segunda línea de los estudios de fragmentación está orientada a la comprensión de la desigualdad social y las barreras materiales e inmateriales entre sectores sociales. Desde esta perspectiva, se interpreta la fragmentación espacial como resultado de un mercado desregulado en la asignación de recursos, en la localización de las inversiones y en la infraestructura urbana, lo que habría derivado en un desarrollo desigual al interior de la ciudad (Rodríguez \& Winchester, 2004). Para algunos, estos procesos también normalizan insistentemente la desigualdad social y la representan como una parte intrínseca de todas las sociedades capitalistas contemporáneas (Soja, 2000).

La fragmentación urbana también se asocia a una ruptura, separación o distanciamiento social en la ciudad, estudiado básicamente mediante la idea de segregación, en tanto coexistencia en la ciudad de grupos sociales distintos que habitan espacios reducidos y separados entre sí (Sabatini, Cáceres \& Cerda, 2001; Rodríguez \& Arriagada, 2004; Sabatini, Salcedo, Wormald \& Cáceres, 2010). .Destacan los autores las diferencias geográficas, en cuanto la localización de los grupos; demográficas, según las características de la población; y sociológicas, en el distanciamiento social en los discursos y conflictos entre estos grupos. Otros autores también mencionan la conformación de una ciudad fragmentada por los discursos del miedo y la seguridad (Dammert, 2004; Souza, 2008; 2008; Caldeira, 2000). Se trata de la fragmentación entendida como una estructura que propicia procesos de segregación o "tugurización", a partir de una cierta "ecología del miedo" (Davis, 1999), que ordena la ciudad en función de un distanciamiento del otro.

La combinación de los factores seńalados da origen a una ciudad fragmentada que involucra componentes espaciales, como la desconexión física y discontinuidades morfológicas; dimensiones sociales, como el repliegue comunitario; y lógicas excluyentes y políticas, como la dispersión de actores y autonomización de dispositivos 
de gestión y regulación urbana (Prévôt-Schapira, 2001). Harvey (2004) estima que está en juego el uso colectivo de la ciudad, la que siempre ha estado fragmentada, aunque nunca dejó de haber relaciones entre los fragmentos y preocupación por unirlos. Hoy, en cambio, se han formado islas o compartimentos estancos, fragmentación que correspondería a una fractura social reflejada en el espacio, que desata las luchas por el derecho a la centralidad (Fani, 2011).

\section{La fragmentación del tiempo}

Sin pretender abordar una discusión sobre las formas ideales de comprensión del tiempo, se plantea la necesidad de incorporar el tiempo social para el estudio de la fragmentación. Frecuentemente se tiende a generar una distinción entre espacio y tiempo de los fenómenos que se estudian, no obstante que estas dos dimensiones acontecen de forma paralela, de manera relacional y procesual. Espacio y tiempo componen las categorías fundamentales sobre las cuales se desarrolla la vida social. Cuando se piensa en el tiempo, se tiende a asociarlo al tiempo cronológico que marcan las agujas del reloj; o, del mismo modo, se piensa en los tiempos largos, que marcan las periodizaciones históricas de carácter lineal. Sin embargo, el tiempo, al igual que el espacio, puede ser pensado como producción social. En esta perspectiva, el tiempo adquiere diversas formas de significación, dependiendo de los contextos sociales, políticos, económicos o culturales a partir de los cuales se esté situado para entenderlo.

La dicotomía, superposición, similitud y yuxtaposición de los conceptos de lugar, espacio y tiempo, ha sido un tema de amplia discusión en el campo de la geografía y los estudios urbanos. En la década de 1970 la geografía del tiempo formulada por Torsten Hägerstrand (1991) explicó el vínculo indisoluble entre tiempo y espacio. La noción de "tiempo-espacio" desarrollada por May y Thrift (2001) también ha sido útil en aprehender los procesos urbanos, al relacionarse con la interconexión del tiempo y el espacio y la multiplicidad de tiempo-espacios. Este concepto intenta superar el dualismo en la comprensión de tiempo y espacio como nociones separadas y las ve como inseparables en su análisis, sin priorizar una sobre la otra.

Complementario con esto, la experiencia del tiempo social es múltiple y heterogénea, varía la cultura entre sociedades e individuos y se relaciona con su posición social. Para esto, May y Thrift (2001) identifican cuatro dominios principales donde el tiempo y el espacio tienen implicancias para las prácticas sociales. En el primero, la experiencia del tiempo-espacio varía según los horarios y ritmos, es decir, según los ciclos diarios, estaciones o ritmos biológicos. Esto puede tener impactos diferenciados en las prácticas sociales, como, por ejemplo, las personas pueden usar distintos modos de transporte según la estación, o los lugares desde y hacia donde viajan pueden ser altamente dependientes de la hora del día. Para el segundo dominio, el tiempo-espacio está formado por sistemas de disciplina social -o, en términos de Lefebvre (2004), ritmos lineales-, incluidos los tiempos del trabajo, del hogar, religioso, de ocio, entre otros, los que tienen variados significados en distintos espacios. Estos tiempo-espacios tienen implicancias en cómo, por qué y dónde las personas se mueven a ciertas horas; por ejemplo, el tiempo para ir a 
trabajar es distinto del tiempo para ir a la iglesia. Un tercer ámbito concierne a la relación con los instrumentos y dispositivos que afectan la forma en que tiempo y espacio se relacionan con la práctica social. Los teléfonos móviles o los computadores han generado que la movilidad física sea menos necesaria; asimismo, el uso de medios de transporte más rápidos tiene implicancias relevantes en la forma en que se vive el tiempo-espacio. Finalmente, el tiempo-espacio se traduce en varias formas de representación, las cuales requieren comprensión de las prácticas que se llevan a cabo, incluyendo patrones, experiencias y condiciones de viaje.

En el contexto de la globalización, se ha analizado la compresión del tiempo por medio de la conquista del espacio (Santos, 1994; Harvey, 1994; 2004). En estas argumentaciones, las distancias que recorren los flujos de información, dinero, bienes y personas han sido superadas por medio del desarrollo de tecnologías de transporte y comunicaciones. Esta idea ha sido interpretada de forma apresurada por las ciencias sociales, que han argumentado la muerte del espacio ante la conquista del tiempo (Haesbaert, 2007), noción que contrasta de forma notoria con la realidad de las ciudades, donde gran parte de las personas debe enfrentar a diario barreras físico-espaciales para desarrollar sus actividades, haciéndolo por medio de prácticas de movilidad cotidiana (Jirón \& Mansilla, 2013). Es importante considerar que la globalización no ha eliminado la importancia del espacio y del tiempo en la vida cotidiana, sino que, muy por el contrario, ha generado impactos divergentes sobre la experiencia espacio-temporal (Massey, 1995; 2005). En este sentido, la fragmentación espacial que hoy reconfigura el espacio urbano ha incidido de manera considerable sobre diversas formas de fragmentación del tiempo en la sociedad (Jirón, 2010a, 2010b; Jirón, Lange \& Bertrand, 2010; Jirón \& Mansilla, 2013; Lange, 2011; Cebollada, 2009; Gutiérrez, 2009; Jarvis, 2005).

A partir de los resultados obtenidos en esta investigación se reconocen tres formas posibles de fragmentación espacio-temporal en la sociedad.

En primer lugar se encuentra la vinculación entre fragmentación espacial y fragmentación temporal y sus impactos en la vida cotidiana. Tal como lo menciona Salvador (2000), "los habitantes de las ciudades funcionalizan cada vez más su vida cotidiana, dividiéndola espacial y temporalmente, en actividades cada día más básicas por más especializadas" (p. 123). La existencia de un espacio discontinuo y atomizado ha determinado una experiencia temporal fragmentada de quienes transitan en la ciudad, que adaptan su uso del tiempo mediante estrategias para unir las partes fragmentadas de la realidad (Jirón, 2010a).

La segunda se refiere a la fragmentación existente entre las condiciones apropiadas de uso del tiempo y aquellas imposiciones temporales que la globalización, las instituciones y la sociedad de consumo asignan a nuestros ritmos en la ciudad (Lefebvre, 2004; Fani, 2011; Salvador, 2000). Los tiempos y ritmos que se imponen son disonantes respecto de los tiempos de las personas, lo que las obliga a programar a diario sus vidas, estableciendo estrategias para resolver las barreras temporales impuestas socialmente. Los tiempos de la vida cotidiana se encuentran segmentados y resquebrajados: los tiempos de la familia, de comer, de compartir, son eliminados en pos de resolver el programa de actividades impuesto. 
Una tercera forma de fragmentación acontece cuando la distinción del tiempo cronológico como totalidad se desvanece ante las posibilidades diferenciales que los grupos sociales poseen para lograr la compresión espacio-temporal. Solo algunos grupos sociales pueden acceder a la hipermovilidad facilitada por las tecnologías de las comunicaciones y del transporte, mientras otros se encuentran inmovilizados o siguen moviéndose en los ritmos lentos, marcados por la precariedad (Santos, 1994; Mansilla, 2011b), insertos en túneles de separación que tienen espesuras, espacios, frecuencias, duración, entornos, calidades, altamente diferenciados (Jirón, 2010b).

Si bien estas consideraciones no representan todas las formas de fragmentación temporal, permiten demostrar que efectivamente existe una fragmentación entre las experiencias y las temporalidades impuestas por el sistema económico actual. A partir de estas ideas es posible proponer una aproximación a la fragmentación del tiempo como la constitución de una experiencia temporal diferenciada, determinada por las condiciones materiales de la ciudad y, al mismo tiempo, por las condiciones socioculturales de quienes la habitan, generando túneles diferenciados de temporalidad en movimiento.

\section{La movilidad como enfoque para el estudio de la fragmentación espacio-temporal}

Frente a esta ruptura intencionada de las formas contemporáneas de hacer ciudad, a partir de un enfoque de movilidad cotidiana se devela que los habitantes responden de mejor o peor manera a moverse para acceder a las actividades, relaciones sociales y lugares que les interesan. En el presente estudio esto se enfrenta en términos teóricos desde la movilidad y la vida cotidiana.

El enfoque de movilidad cotidiana no solo hace referencia al desplazamiento físico de las personas entre un espacio y otro en la ciudad, sino que se refiere a las consecuencias sociales, culturales, económicas, físicas de este desplazamiento. Este enfoque resulta fundamental para entender la relación entre sociedad y tiempoespacio, ya que la movilidad es un elemento dominante y constitutivo del vivir contemporáneo y del espacio urbano. Al examinar de cerca estas experiencias, las ideas de fijación, permanencia y dualidad presentes en la mayor parte de los análisis urbanos se comienzan a cuestionar y las experiencias móviles emergen como procesos fluidos y multiescalares en su complejidad situada. Esta manera de analizar las prácticas es parte del giro de la movilidad (Urry, 2007; Cresswell, 2006), que está permitiendo avances considerables en términos teóricos, metodológicos y prácticos para comprender las sociedades contemporáneas.

El enfoque de movilidad aquí propuesto incorpora al habitante desde su vida cotidiana en movimiento, para develar fenómenos urbanos que resultan imperceptibles desde otros enfoques. La vida urbana contemporánea se encuentra cada vez más influenciada por la movilidad, sea esta física, virtual o imaginada. Del mismo modo, la no movilidad, o la movilidad restringida, puede ser uno de los aspectos que más certeramente reflejan las desigualdades que se viven en nuestras ciudades. 


\section{El urbanismo fragmentador}

En los análisis de la fragmentación urbana descritos de manera previa se evidencia una visión estática y dual de la ciudad, donde se da cuenta de la localización de las intervenciones y la separación entre estas, pero poco se menciona respecto del dinamismo e interrelación entre segmentos, el movimiento constante en que viven los habitantes de ciudades y la manera en que se ven afectados por cada nueva intervención urbana.

En este contexto, denominamos urbanismo fragmentador a aquella práctica de intervención urbana (pública y privada) que fragmenta la vida cotidiana de los habitantes urbanos. Desde esta perspectiva, más que un análisis morfológico, funcional, locacional o estructural del espacio, lo que se intenta destacar es la manera en que los habitantes se ven afectados por esta "ciudad fragmentada" y, fundamentalmente, cómo enfrentan esa fragmentación en la cotidianidad.

Frente a las intervenciones de nuevas áreas productivas o de servicios en la ciudad - malls, autopistas urbanas, barrios cerrados, entre otros-, los habitantes urbanos no permanecen inmóviles, presos en sus barrios. Muy por el contrario, se desplazan de manera constante por la ciudad, relacionándose con otros y estableciendo vínculos entre los fragmentos que se generan.

Desde una mirada de la movilidad, se reconoce a lo menos tres formas de intervenciones urbanas que crecientemente fragmentan la experiencia urbana. La primera se relaciona con las estructuras discontinuas y la extensión urbana, producto de la expansión inmobiliaria y el despliegue de megainfraestructuras que han incidido de forma considerable sobre los procesos de fragmentación físico-espacial de la ciudad. La segunda es la automovilización de la ciudad, promocionada por políticas públicas de desarrollo urbano por medio de la inversión público-privada en sistemas de autopistas que potencian el uso del automóvil, así como producto de un sistema de transporte público deficiente en ciertas áreas de la ciudad en términos de conectividad, accesibilidad y frecuencia, fenómeno que puede ser evidenciado también por el incremento constante del parque automotor en las grandes metrópolis. La tercera forma de intervención se relaciona con la mala calidad de las intervenciones urbanas marcadamente diferenciadas según zonas de la ciudad, tanto en los barrios como en las diversas etapas de los trayectos, acentuando condiciones de precariedad en la experiencia del viaje e imponiendo barreras para el desplazamiento. Las intervenciones, cuando se realizan en áreas segregadas de la ciudad, son generalmente de baja calidad; y cuando se interviene con alta calidad, la intervención es puntual, aislada, para el uso de ciertos destinatarios.

La forma en que se interviene la ciudad a partir de un enfoque estático, que no reconoce las implicancias cotidianas de las intervenciones, efectivamente genera una fragmentación en su estructura. Esta forma de actuar sobre las ciudades no solo genera territorios morfológicamente desmembrados, fracturados, separados y divididos, sino que, además, fragmenta las condiciones espacio-temporales de la vida cotidiana de las personas.

En metrópolis como Santiago, los habitantes ocupan no solo los barrios en que viven; sobre todo los residentes de áreas segregadas viven su cotidianidad utilizando el resto de la ciudad. Precisamente debido a la carencia de infraestructura y servicios 
básicos (salud, educación, recreación, comercio), insuficientes fuentes de empleo y calidades espaciales deficitarias en dichos territorios, las personas se ven obligadas a salir diariamente de los sectores residenciales para acceder a tales servicios y así enfrentar lo que la ciudad dispone para ellos. Esto genera circuitos diferenciados en la ciudad, a modo de túneles para los distintos grupos sociales, que se superponen, se traslapan y pocas veces se encuentran.

Graham y Marvin (2001) plantean que por medio de los flujos y nodos de intersección del splintering urbanism (urbanismo astillador), los habitantes comunes y corrientes tejen cotidianamente los fragmentos de la ciudad que este proceso deja detrás, para poder zurcir sus vidas en maneras más o menos coherentes. Lo hacen por medio de la implementación de estrategias de movilidad cotidiana, como llevar a los nińos al colegio camino al trabajo, dormir en los buses, usar transporte público para acudir a los diversos trabajos, encontrarse con amigos en el metro, depender de las redes sociales para sobrellevar la manera fragmentada en que la ciudad está siendo construida.

\section{Consecuencias del urbanismo fragmentador}

Con el fin de aprehender las experiencias de movilidad, la investigación en la que se basa el presente artículo adoptó un método etnográfico, debido a que permite adentrarse en la experiencia cotidiana de los viajeros de una manera mucho más profunda que la mayoría de los métodos cualitativos existentes. El trabajo fue realizado en la ciudad de Santiago en los años 2010 y 2011, período donde se acompañó durante sus desplazamientos cotidianos a diversos viajeros urbanos de ingresos bajos, medios y altos que habitan distintos sectores de la ciudad. Se utilizó el método de "sombreo" (Jirón, 2010c), consistente en un acompañamiento a los participantes en la investigación de manera individual en sus rutinas cotidianas, donde el investigador observa la manera en que llevan a cabo su práctica, a modo de sombra. Ello implica acompañar a los participantes en sus trayectos diarios desde el momento en que salen de su hogar hasta que vuelvan y consideren que sus viajes han terminado por el día.

Dependiendo de la situación, el investigador puede discutir temas durante el periodo de sombreo o mantenerse en silencio, mientras los participantes saben que están siendo sombreados. El viaje puede ser fotografiado, grabado (sonido o video). Técnicas similares han sido desarrolladas en la investigación fenomenológica de las experiencias espaciales urbanas, incluyendo los "acompañamientos" de Kusenbach (2003) o Spinney (2009) o los diarios de viaje de Latham (2004). La metodología aquí desarrollada ha probado ser adecuada para capturar experiencias de movilidad cotidiana de individuos en la ciudad de Santiago (Jirón, 2010c), debido a su flexibilidad y posibilidad de penetrar, por medio de una descripción profunda y multifacética, en las rutinas diarias de habitantes urbanos móviles.

El principal producto de este seguimiento es el relato etnográfico, utilizado aquí como dato de análisis. En este sentido, los relatos etnográficos y su análisis no se centraron exclusivamente en los viajes durante los cuales las personas fueron acompañadas, sino que integraron en la narración el contexto en el cual estos viajes se 
sitúan: las explicaciones que las personas generan respecto de sus decisiones; sus quejas, frustraciones, ideas sobre cómo podría mejorar su forma de moverse y su vida en general; los preparativos para el viaje, los acuerdos con otros que facilitan o permiten la realización de esos viajes, obstáculos en su organización cotidiana o respecto de sus expectativas, las comparaciones con el pasado propio y ajeno en las formas de moverse, recuerdos gatillados por las conversaciones o lugares durante los viajes, entre otros aspectos.

Los casos incorporados al estudio se definieron en tres etapas. En la primera se dividió la ciudad de Santiago según el dinamismo de flujos en la ciudad utilizando los datos de la Encuesta de Movilidad del Gran Santiago, del 2006 (Secretaría de Transporte [sectra], 2006) y el Censo 2002 (Instituto Nacional de Estadísticas [INE], 2002), por medio de los cuales se definieron las zonas de dinamismo según centro, pericentro, periferia y extraperiferia, y se seleccionaron comunas de la ciudad que correspondían a estas zonas. En una segunda etapa se seleccionaron sectores dentro de las comunas representativas de estas zonas donde se pudiese encontrar hogares de ingresos bajos, medios y altos, según correspondiese; en algunas zonas solo se pudo seleccionar sectores de ingresos bajos, en otros solo altos y en algunos los tres rangos. Finalmente se seleccionaron miembros de hogares que tuvieran una movilidad activa (salir de su casa todos los días) y que correspondieran a diversos grupos: hombres y mujeres, de diversas edades e ingresos. Con estos criterios se sombrearon 76 casos en ocho zonas de Santiago (Santiago centro, El Golf/ Sanhattan, La Cisterna, Huechuraba/Conchalí, Las Condes, Maipú, La Florida, Puente Alto, Talagante y Colina).

A continuación se presentan dos casos de los seleccionados, los que corresponden a un viajero de ingresos bajos y uno de ingresos altos; el primero vive en la extraperiferia de la ciudad y el segundo en la periferia, división según el dinamismo de flujos en la ciudad de Santiago. Estos casos se seleccionaron para este trabajo por sus diferencias significativas de experiencia de viaje, ya que sus relatos dan cuenta de manera bastante clara de situaciones que se repiten en distintos casos estudiados. Por otro lado, se seleccionaron por provenir de áreas marcadamente de distinto nivel de ingreso, para así dar cuenta de las diferencias que se perciben al viajar. Por medio de estos casos se puede observar cómo, pese a las dificultades que presenta la ciudad, los viajeros ineludiblemente se desplazan por ella, con menor o mayor grado de dificultad.

El primer caso describe la experiencia cotidiana de viaje de Pablo, quien vive en Colina, comuna ubicada en la extraperiferia del norte del Gran Santiago, la que ha sufrido diversas transformaciones durante las últimas décadas. De ser una comuna rural ha pasado a ser una comuna rururbana, con edificaciones de lujo, producto de un amplio incentivo al desarrollo inmobiliario durante la última década e inversiones privadas en autopistas que unen la periferia urbana con las nuevas áreas centrales de la ciudad, aunque manteniendo un porcentaje alto de viviendas de bajo valor. Pablo viaja todos los días a Santiago usando diversos medios de transporte, y zurce los pedazos de la ciudad pese a las variadas dificultades que se presentan.

El segundo caso es Simón, viajero de al tos ingresos que vive en Las Condes, comuna pericentral en el oriente del Gran Santiago, que se destaca en el ámbito nacional por 
estar entre las de mayor nivel de ingresos económicos, ser sede de una gran cantidad de edificios corporativos y albergar a los estratos socioeconómicos más altos del país. Se ha configurado así como el nuevo centro de negocios y servicios en Santiago de Chile, integrando diversos tipos de modelos de desarrollo urbano y arquitectónico de escala global. Del mismo modo, la gran cantidad de recursos económicos con los que cuenta la comuna permiten el desarrollo de múltiples inversiones urbanas por parte del municipio, que pretenden integrar a futuro -por ejemplo- sistemas de transportes propios, como trenes en superficie. Simón viaja en automóvil, no se entera de la fragmentación: para él es que está construida esta ciudad.

\section{Pablo cruza la ciudad en transporte público}

Pablo reside en Colina desde que tiene memoria. Tiene cuarenta y dos años y vive con su hijo de ocho años y su esposa en la población Cordillera Iv. Sale a las 5:30 de la mañana de su casa mientras está oscuro aún, pero ya hay gente en los paraderos de Colina. Dice que por atrás pasa una micro que le queda más cerca, pero que tiene una frecuencia de 30 minutos, por lo que prefiere caminar un poco más e irse a la segura con la locomoción. Cuenta que el servicio en Colina es bastante irregular, que hay tres tipos de locomoción de un mismo dueño, cada una con una tarifa distinta -800 , 900 y 1.000 pesos-, dependiendo de la calidad y rapidez con la que se llega a Santiago. Al parecer, hasta las 6:30 de la mańana la locomoción es expedita, pero desde esa hora en adelante ya es imposible irse sentado hasta Santiago.

La micro se hace pequeña, es para unas 25 personas sentadas. Pablo comienza a acomodarse para hacer el viaje durmiendo. De a poco, empiezan a asomarse industrias, sale humo de una fábrica y la micro se detiene para recoger a un pasajero en la autopista, mientras el traslado continúa en absoluto silencio. Pasan frente a tiendas de marca, como Nike y Construmart; a lo lejos se alcanza a ver un bus alimentador del Transantiago en una avenida próxima. Al parecer, están acercándose a la capital. Llegan a avenida Vespucio y dos pasajeros se bajan; cada vez que el bus se detiene, entra el frío helado por la puerta. La autopista da paso a las calles, comienzan a aparecer los autos, otras micros, hay mayor iluminación y un ruido que se intensifica hasta llegar a Mapocho, el centro de Santiago.

Todos los pasajeros descienden, pese a que el terminal está a unas cuadras; pero esta parada es mejor para bajarse, ya que queda más cerca del metro. El aire está helado y todos están abrigados. La vereda es muy angosta y Pablo comenta que le resulta incómodo caminar por esta parte de la orilla del río, debido a su cojera. La vereda, además, muestra bastantes imperfecciones, lo que implica el riesgo de dar pasos en falso y tropezar. Ya hay gente en el paradero donde pasa locomoción hacia la Alameda. Pablo baja a la estación del metro a las 6:35 y avanza hasta el andén. Entra al vagón y se queda de pie; no vale la pena sentarse, pues recorrerá apenas un par de estaciones. A los pocos minutos llega a la estación Rondizzoni, su destino.

Pablo sale del metro esquivando a los que vienen entrando, pasa a la boletería a cargar de dinero su tarjeta de pago del transporte y aprovecha de cambiar monedas. Cerca de su trabajo no hay dónde cargarla y si no lo hace ahora, a la vuelta la tarjeta no tendrá fondos suficientes para pagar el viaje. Todavía está oscuro y ya se ven más 
vehículos transitando por la autopista. Camino al paradero, Pablo pasa a comprar leche chocolatada y un galletón.

Pablo camina a paso tranquilo y se abriga un poco más. Llega al paradero y hay seis personas esperando. Pronto pasa la micro 101. Adentro hay alrededor de seis personas, solo dos son mujeres, casi todos van despiertos y dos hombres van comiendo. Pablo comienza a tomar su leche y abre el paquete del galletón. Dice que se la toma en la micro porque no está seguro de poder hacerlo tranquilo en el trabajo, una empresa de metalurgia. Pese a que la jornada empieza a las 8:00, Pablo llega a las 7:30 para tomar desayuno, arreglarse y pagar menos en la "hora valle" del Transantiago.

El bus bordea la Autopista del Sol; al frente se alcanzan a ver murallas rayadas y al rato se entra en un sector industrial en el que hay prácticamente solo fábricas. Al bajarse en Cerrillos, el aire está más helado. Camina unas tres cuadras largas, las luces ya están encendidas en los galpones por los que pasa y en algunos ya sale humo. Se detiene frente a una reja gris desde donde se alcanza a observar un galpón y algunos tarros; al lado hay una cabina de guardias vacía y cerrada. "El dueño dice que le sale muy caro tener un nochero, así que tenemos que esperar a que llegue el hijo del dueño para que nos abra, lo que es relativo, dependiendo de la hora en la que se levante", dice Pablo, mientras espera frente a la reja, junto a otro compańero, veinte minutos hasta que el encargado llega en una gran camioneta.

El caso de Pablo permite evidenciar la forma en que transita por las autopistas, para acceder desde el fragmento espacial del barrio en el que reside, al fragmento del trabajo. Su temporalidad se encuentra dominada de cierta forma por los tiempos impuestos por las instituciones: por una parte, las del trabajo, que requieren una hora de llegada y un programa de actividades bastante denso; por otra, su movilidad se encuentra condicionada a los tiempos impuestos por las empresas de buses que mantienen el control monopólico del transporte en la periferia, tanto en términos de tiempos de viaje como en términos de costos. Esto implica formas de fragmentación y de desigualdad espacio-temporal, con posibilidades diferenciadas de acceder a la compresión temporal.

El patrón de viaje es rutinario y estructurado. Existen pocas posibilidades de salirse de un ruta predefinida, o de combinar con otras formas de movilidad. En este sentido, las posibilidades de movilidad de Pablo son reducidas, ya que la organización del transporte y del espacio urbano por el que transita no entrega suficientes alternativas, afectando incluso en términos de dificultades de desplazamiento.

La experiencia de la fragmentación, en el caso de Pablo, se hace presente también mediante deslocamientos espacio-temporales. Ello ocurre cuando desarrolla al interior del bus y del metro ciertas prácticas que comúnmente deberían ser ejercidas en otros espacios de su vida cotidiana, vinculados a esa funcionalidad. Por ejemplo, Pablo debe comer arriba del bus o del metro, ya que el programa de actividades impuestas por el trabajo no le permiten tener seguridad de que podrá hacerlo posteriormente. También el levantarse con mucha anticipación para llegar al trabajo a la hora, el tener poco tiempo libre, acentúan las condiciones de fragmentación del tiempo y del espacio.

Existen variaciones considerables en los ritmos en los cuales Pablo tiene que insertarse para moverse en la ciudad. Así, por ejemplo, intenta salir más temprano, 
cargar dinero en la tarjeta con que paga el transporte y generar una serie de estrategias que le permitan sortear las dificultades que pueden presentarse en el viaje. Los instrumentos y los dispositivos de movilidad parecen no estar bajo el control de Pablo. Es él quien tiene que adaptarse a las condiciones definidas por los instrumentos y dispositivos de movilidad que se encuentran dispuestos.

\section{Simón optimiza su tiempo}

Simón tiene cuarenta y dos años, está casado con Rosario, tienen dos hijos (Diego e Ignacio) y viven en una casa en Las Condes. Es diseñador y propietario de una oficina de diseño ubicada en Sanhattan, el nuevo barrio de negocios de Santiago, donde trabaja con su esposa. Su barrio es arbolado, con grandes casas enrejadas; en su casa hay cuatro automóviles estacionados: un Citroën Picasso, un BMw descapotable blanco, un Smart azul y una camioneta Ford 4x4, junto a tres motos, cubiertas con fundas plásticas.

Sube al Citroën y su hijo Diego conduce, ya que está aprendiendo a manejar. La radio está prendida. El auto se encuentra ordenado y limpio, huele a nuevo. El camino es expedito, sin tacos ni demoras, considerando esperas en semáforos. Pronto se presenta un taco: padres que dejan a sus hijos e hijas en la puerta del colegio, sumados a vehículos de trasporte escolar detenidos mientras bajan estudiantes, generan atochamiento. El tránsito se transforma en un flujo lento, pero constante. Diego baja del vehículo, se despiden de beso y Simón toma la conducción. Se detiene en una esquina. "Este semáforo rojo es eterno", dice.

Simón reconoce que al ser el dueńo de su empresa maneja su propio horario, pero "no paro en todo el día, estoy todo el tiempo trabajando". Al transitar se encuentra con atochamientos; sin embargo, agrega "en media hora más esto es un tremendo taco", mientras se desplaza por la calle Presidente Riesco. También dicta clases en un Instituto. "Estoy en la pará de pasarlo bien, no estoy preocupado del costo-beneficio, soy el motor de mi empresa”, dice, sosteniendo que le gusta trabajar con flexibilidad horaria, ya que le permite desarrollar la veta académica, donde forma a estudiantes que luego trabajarán para él.

Dobla por la calle Augusto Leguía e ingresa al estacionamiento subterráneo del Hotel W. Llega a su oficina diez para las ocho. A las 14:10 se pone la chaqueta y sale en dirección al Instituto a las 14:15. Al llegar al estacionamiento, abre otro auto, el вмw descapotable blanco en que llegó su esposa, estacionado detrás del Citroën en que llegó él por la mañana. Se sube rápido mientras comenta "estamos atrasados, pero el cliente no puede saber eso; yo debo actuar de forma natural, como si nada pasara”. Sale por la calle Augusto Leguía, suena su iPhone y contesta con total naturalidad mientras conduce. Pese a que la conversación fue corta, él sigue manipulando su iPhone.

No usa el transporte público. Dice: "No recuerdo la última vez que anduve en micro, pero tengo una tarjeta Bip!", la misma de pago del transporte público que sí usa Pablo. Esto se debe en principio a que no tiene tiempo para perder, debe desplazarse rápida y cómodamente por la ciudad, despreocupándole el costo que ello implica. A las 14:30 llega al Instituto donde hace clases y tiene una reunión almuerzo hasta las 15:30. 
Tras pedir la cuenta, se despide y se dirige al auto. Conduce por la calle Andrés Bello. Para frente a un semáforo en rojo, toma su teléfono y lo utiliza, y agrega "el auto sigue siendo como mi oficina". Comenta que dicha conducta es habitual, "me han sacado más de un parte, lo asumo y lo seguiré asumiendo". Estaciona el vehículo y se dirige al ascensor.

El caso de Simón destaca en comparación con los casos anteriores, ya que su posición social y económica le permite acceder a ciertos fragmentos espaciales con mucha más facilidad que a otras personas. Sin embargo, cuando se analiza su trayecto cotidiano es posible evidenciar que su movilidad también se encuentra restringida a un circuito de fragmentos.

Simón accede a un tiempo liso, la fragmentación temporal es menor, ya que tiene un control total sobre los dispositivos de movilidad, por sus posibilidades de acceso tanto al automóvil como a tecnologías de la comunicación, que le permiten manejar diversos fragmentos espaciales de la ciudad donde él puede estar presente o por donde puede desplazarse de manera rápida, sea física o virtual. Sin embargo, reconoce que los cambios de horarios marcan claramente diversos ritmos en la experiencia de viaje en la ciudad. Pese a que tiene flexibilidad en términos de horario por ser su propio jefe, la fragmentación temporal se encuentra dada por la gran cantidad de actividades que debe desarrollar en un día, y por algunas limitaciones horarias menores que impone la ciudad para el desplazamiento, a causa de la planificación de las vías de transporte. Existe un aprovechamiento total del tiempo, dice no poder "perder tiempo".

El caso de Simón da cuenta de una persona que logra acceder a la hipermovilidad mediante una estrategia que comprende un deslocamiento de la experiencia espacial, donde "la oficina es el auto".

\section{Consideraciones sobre la movilidad en Santiago de Chile}

Por medio de este estudio es posible detectar el impacto que genera el tipo de urbanismo que prevalece en la ciudad y las estrategias que las personas desarrollan para zurcir su vida a lo largo de ella. A partir de esto se pueden generar algunas consideraciones generales en relación con las debilidades teóricas del concepto de fragmentación mencionadas al inicio.

En ciudades como Santiago, la posibilidad de estar confinado no se aplica solo a áreas fijas, como el trabajo o el hogar, sino que también puede ocurrir en movimiento. Esto se debe a las largas distancias y extensas horas de viaje diario, acompańadas por experiencias de movilidad monótonas y difíciles, lo que puede llegar a que algunos, a medida que van zurciendo los espacios fragmentados, tengan que "saltarse" la ciudad al trasladarse por circuitos en forma de túneles. Ajenos a su entorno, otros pueden escoger viajes expeditos e ininterrumpidos, también cobijándose en túneles por la ciudad.

La ciudad se restringe de maneras específicas, creando túneles determinados dependiendo de las características y posibilidades sociales. Estos son espacios de homogeneidad, no necesariamente de integración, y en general de aislamiento. Cada túnel se vuelve una estrategia para evadir al otro, pasando cerca de él, pero 
sin encontrarse. Esta situación se ve exacerbada cuando el miedo, el tráfico o la posibilidad de tener un automóvil se unen. La posibilidad de saltarse la ciudad en un túnel más sofisticado, como un automóvil, se encuentra disponible para aquellos con mayores recursos y flexibilidad.

Pese a que en los casos descritos anteriormente la condición socioeconómica es la que más diferencia la manera en que los viajeros unen o zurcen su ciudad, es posible identificar otras condiciones socioculturales (edad, ciclo de vida, etnia, religión, etcétera) que pueden incidir en la forma de unir las partes fragmentadas de la ciudad. En los casos presentados se puede detectar factores que, desde la experiencia de viaje, hacen que cada túnel sea más o menos placentero para el viajero. Por ejemplo, se pueden identificar factores de comodidad, calidades espaciales, paisaje, flexibilidad y disponibilidad de opciones, el saber viajar o desarrollo de estrategias.

En cuanto a lo desagradable, Pablo experimenta situaciones incómodas en su viaje -ir apretado en el bus, sentarse en asientos incómodos, transitar por veredas demasiado angostas-, mientras que Simón viaja con alto nivel de comodidad en su automóvil. Las calidades espaciales también se diferencian, desde los lugares por donde Pablo transita, con suciedad, en mal estado, paraderos incómodos o inexistentes o calles sin pavimentar, a las calles arboladas y bien mantenidas de Simón. Esto lleva a reconocer las diferencias paisajísticas por las que transitan estos viajeros. Pablo, que cruza parte importante de la ciudad, percibe desde su ventana industrias, carreteras, basurales, mientras que el paisaje que observa Simón es de mayor prolijidad estética y atractivo.

Sus viajes, sin embargo, se diferencian no solo por las condiciones físicas del mismo, sino también por la flexibilidad que cada uno puede tener. Los viajes de Pablo están restringidos por los horarios de trabajo, disponibilidad de transporte público y capacidades individuales. Además de esto, su lugar de residencia se encuentra lejos de su trabajo, y la ausencia de Transantiago en Colina lo obliga a pagar un alto costo de transporte para llegar a su trabajo. Simón, por el contrario, está inserto en un sistema de gran flexibilidad y múltiples opciones, que cubren desde los modos de transporte, rutas, horarios, formas de trabajo, estacionamientos, hasta la música que escucha. Esto hace su viaje más fácil y agradable.

Finalmente, en cuanto al conocimiento que cada uno tiene de su viaje y del trayecto específico, los dos casos presentan estrategias diversas para moverse de la mejor manera posible. Se puede identificar varias de las circunstancias que los viajeros de transporte público, como Pablo, han tenido que aprender a dominar: los horarios del transporte, los lugares por donde transitar, donde pararse, donde bajarse, cómo poner su cuerpo en los asientos, cómo comportarse con el extrańo, en qué momento es mejor comer o dormir o bajarse. Lo mismo para Simón: el tiempo es su gran recurso escaso, y sabe cómo moverse, por dónde viajar, y está dispuesto a enfrentar un parte o pagar peajes, si es necesario, para mejorar su estrategia de viaje.

Estos factores muestran que, pese a la importancia de intervenir en mejoras en la morfología o funcionalidad de la ciudad, también pueden existir otros factores que incidan en mejorar la forma de zurcir la vida cotidiana. Ello indica que es necesario comprender las externalidades que generan los procesos de urbanización y que, a veces, una mejora en calidad de vida de algunos incide en la calidad de vida de otros. Reconocer estas implicancias resulta importante hoy, ya que estos tiempo-espacios 
túneles que se ven cada vez más excluidores y restringidos, pueden devenir en oportunidades, si se aprovechan. Es decir, que pese a que algunas personas pueden sentirse confinadas en lugares fijos, la experiencia de movilidad podría generar la posibilidad de expandir lugares durante o por medio del viaje, permitiendo acceso a nuevas y diferentes actividades, relaciones, lugares y objetos en la ciudad.

\section{Conclusiones}

El urbanismo fragmentador comprende una práctica institucionalizada que genera severos impactos sobre la vida cotidiana de los habitantes de la ciudad. Superar dicha práctica implica incurrir en la búsqueda de nuevas formas de estudiar e intervenir la ciudad, para ello se requiere de un giro epistemológico que integre en toda su complejidad la relación espacio-tiempo-sociedad.

En este sentido, los diversos sesgos en que han incurrido los estudios urbanos tradicionales al tratar el tiempo y el espacio como categorías neutras, han contribuido a la formalización de una práctica urbanística que interviene sin considerar la complejidad espacial que encierra su acción. Se puede encontrar claros ejemplos de esta situación en los estudios enfocados en describir los procesos de fragmentación y segregación en la ciudad. La discusión de los antecedentes hasta aquí expuestos permite considerar la necesidad de incorporar en este giro epistemológico a lo menos tres aspectos: i) el espacio y el tiempo como producciones sociales, ii) las prácticas móviles de la sociedad que generan interacción entre los fragmentos en que ha sido descompuesta la ciudad, y iii) el rescate de las formas de apropiación espacial de los sujetos, mediante sus prácticas y significaciones.

La primera consideración, repensar el espacio y el tiempo como producciones sociales, evoca la necesidad de plantear que más allá de la racionalidad instrumental que mantienen los planificadores urbanos y las intervenciones tecnócratas de los urbanistas, espacio y tiempo representan producciones sociales. Es decir, son el resultado de procesos políticos, económicos y sociales y, por tanto, cualquier tipo de intervención tendrá impactos divergentes sobre cada uno de esos ámbitos. Las nociones de espacio y tiempo cambian al ritmo de las formas de apropiación y significación espacial que los sujetos y los grupos sociales generan de ellos. Por tanto, existen múltiples formas de producción de espacio en la ciudad, que la mayoría de las veces se encuentran en conflicto con los intereses de los urbanistas que planifican y proyectan la ciudad a partir de las necesidades de las corporaciones privadas, borrando cualquier forma alternativa de reproducción de la ciudad que surja desde el habitar.

La segunda consideración sobre la movilidad se encuentra vinculada también a la carencia de una aproximación a la comprensión de la dimensión espacio-temporal. A pesar de que ambas dimensiones son indisolubles, el urbanismo fragmentador ha tendido a su disociación, enfocado principalmente en describir las transformaciones espaciales, en desmedro de la dimensión temporal. Esto representa una debilidad no menor, debido a que, como ha sido ampliamente discutido en las ciencias sociales, nos encontramos hoy en un periodo en el que el tiempo y la movilidad han cobrado especial importancia en el contexto del paradigma geotecnológico imperante. Estos aspectos nos desafían a abordar la comprensión de la ciudad como un 
espacio dinámico, en constante devenir, donde los flujos de personas, ideas, bienes producen el espacio, rompiendo la imagen estática y atemporal que ha predominado en las representaciones urbanas. La propuesta se centra, así, en la comprensión de un espacio urbano entendido como flujos, más que como áreas.

Por último, es posible argumentar la necesidad de restituir la condición espaciotemporal de los sujetos y los grupos sociales que habitan la ciudad. El urbanismo fragmentador ha descompuesto también la relación sujeto-espacio o sociedadespacio, desconociendo que quienes habitan la ciudad también producen formas de apropiación espacial mediante sus prácticas y significaciones, las cuales comúnmente son desplazadas por el discurso urbanista. Este impone un modelo de ciudad hecho a la medida de los requerimientos de las instituciones y de las corporaciones privadas, que superpone su visión objetivo sobre estas otras formas espontáneas de producción de la ciudad. En esta mirada, los sujetos y los grupos sociales no representan más que una variable demográfica, un simple indicador estadístico que es necesario intervenir para alcanzar la igualdad ajustándola a la media de los datos, o redistribuyendo su localización con el fin de generar mayor heterogeneidad, entendida como sinónimo de igualdad. Esta racionalidad instrumental y tecnocrática sobre la cual se funda el urbanismo fragmentador, borra toda capacidad de acción de los sujetos como partícipes en la transformación de la ciudad. Restituir al sujeto su condición espacial implica generar mecanismos que permitan visualizar las formas en las que las ciudades están siendo creadas y experimentadas por sus habitantes cotidianamente.

Si bien el enfoque de la movilidad no es la única posibilidad para el desarrollo de una nueva epistemología que deconstruya la práctica del urbanismo fragmentador, existen en ella variados aportes que permiten disponer de una herramienta de especial interés para abordar la dimensión espacio-temporal y las formas de exclusión y desigualdad social que experimentan a diario los sujetos en sus prácticas cotidianas en la ciudad. Cualquier propuesta que intente revertir esta racionalidad instrumental debe surgir a partir de un cambio en la posición misma que el investigador mantiene en el plano de las representaciones, eliminando la observación distante, que interviene la realidad sin estar presente en ella. Es necesario invertir el orden de la investigación, eliminando las interpretaciones teóricas y metodológicas a priori, cambiándolas por un conocimiento que se construye a partir de la multiplicidad de experiencias que surgen en la ciudad.

Finalmente, la reflexión de este artículo nos conduce a abrir un debate necesario sobre los impactos que la racionalidad instrumental del urbanismo fragmentador ha implementado en nuestras ciudades, y muestra la necesidad de generar nuevos estudios para una discusión que amplíe nuestra comprensión sobre la relación espacio-tiempo-sociedad. 


\section{Referencias bibliográficas}

Ascher, F. (2006). Le mouvement dans les sociétés hypermodernes. Texto de la $600^{\mathrm{a}}$ Conferencia "L'Université de tous les savoirs", realizada el 4 de enero de 2004. Video disponible en http://bit.ly/TXCfuc

Augé, M. (2007). Por una antropología de la movilidad. Barcelona: Gedisa.

Borsdorf, A. (2003). Cómo modelar el desarrollo y la dinámica de la ciudad latinoamericana. EURE, 29(86), 37-49. http://dx.doi.org/10.4067/S0250-71612003008600002

Borsdorf, A. (2007). Hacia la ciudad fragmentada. Tempranas estructuras segregadas en la ciudad latinoamericana. Scripta Nova. Revista Electrónica de Geografía y Ciencias Sociales, 7(146/122). En http://www.uibk.ac.at/geographie/publikationen/abstracts/2003. borsdorf.hacia.pdf

Brenner, N. (2001). The limits to scale? Methodological reflections on scalar structuration. Progress in Human Geography, 4(25), 591-614. doi: 10.1191/030913201682688959

Caldeira, T. (2000). City of walls: Crime, segregation, and citizenship in São Paulo. Berkeley, Ca: University of California Press.

Caprón, G. \& González, S. (2006). Las escalas de la segregación y de la fragmentación urbana. TRACE, 49, 65-75. En http://biblat.unam.mx/es/revista/trace-mexico-d-f/3

Caravaca, I. (1998). Los nuevos espacios ganadores y emergentes. EURE, 24(73), 5-30. http:// dx.doi.org/10.4067/S0250-71611998007300001

Cariola, C. \& Lacabanca, M. (2001). La metrópoli fragmentada: Caracas entre la pobreza y la globalización. EURE, 27(80), 9-32. http://dx.doi.org/10.4067/S025071612001008000002

Cebolleda, A. (2009). Mobility and labour market exclusion in the Barcelona Metropolitan Region. Journal of Transport Geography, 17(3), 226-233. http://dx.doi.org/10.1016/j. jtrangeo.2008.07.009

Corrêa, L. (1989). O espaço urbano. São Paulo: Editora Atica.

Corrêa, L. (2006). O urbano e a cultura alguns estudos. En R. L, Corrêa \& Z. Rosendal (Orgs.), Cultura, espaço e o urbano (pp. 141-165). Río de Janeiro: Editora da Universidade do Estado do Rio de Janeiro (EdUERJ).

Cresswell, T. (2006). On the move. Mobility in the Modern Western World. Nueva York: Routledge.

Dammert, L. (2004). ¿Ciudad sin ciudadanos? Fragmentación, segregación y temor en Santiago. EURE, 30(91), 87-96.http://dx.doi.org/10.4067/S0250-71612004009100006

Davis, M. (1999). Ecology of fear: Los Angeles and the imagination of disaster. Nueva York: Vintage Books.

De Mattos, C. (1999). Santiago de Chile, globalización y expansión metropolitana: lo que existía sigue existiendo. EURE, 25(76), 29-56. http://dx.doi.org/10.4067/S025071611999007600002

De Mattos, C. (2002a). Mercado metropolitano de trabajo y desigualdades sociales en el Gran Santiago. ¿Una ciudad dual? EURE, 28(85), 51-70. http://dx.doi.org/10.4067/S025071612002008500004

De Mattos, C. (2002b). Transformación de las ciudades latinoamericanas. ¿Impactos de la globalización? EURE, 28(85), 5-10. http://dx.doi.org/10.4067/S025071612002008500001 
De Mattos, C. (2006). Modernización capitalista y transformación metropolitana en América Latina: cinco tendencias constitutivas. En A. I. G. Lemos, M. Arroyo \& M. L. Silveira (Eds.), América Latina: cidade, campo e turismo (pp. 41-73). Buenos Aires: Consejo Latinoamericano de Ciencias Sociales (CLACso), Universidad de São Paulo.

De Mattos, C. (2010). Globalización y metamorfosis metropolitana en América Latina: De la ciudad a lo urbano generalizado. Revista de Geografia Norte Grande, 47, 81-104. http:// dx.doi.org/10.4067/S0718-34022010000300005

Ducci, M. E. (2002). Área urbana de Santiago 1991-2000: Expansión de la industria y la vivienda. EURE, 28(85), 187-207. http://dx.doi.org/10.4067/S0250-71612002008500010

Elgueta, M. (2006). Gran Santiago: Transformación del patrón espacial de localización del sector residencial de la elite. Memoria para optar al Título Profesional de Geógrafo, Facultad de Arquitectura y Urbanismo, Universidad de Chile. En http://www.tesis.uchile.cl/ tesis/uchile/2006/elgueta_m/html/index-frames.html

Elliott A. \& Urry, J. (2010). Mobile lives. Nueva York: Routledge.

Escolano, S. (2007). Cambios recientes de las estructuras espaciales de las grandes ciudades: fragmentación física, segregación socioeconómica y reorganización funcional. En Artigues et al. (Eds.), Los procesos urbanos postfordistas. Actas del viII Coloquio y Jornadas de Campo de Geografía Urbana. Grupo de Geografía Urbana de la Asociación de Geógrafos Españoles (AGE), Islas Baleares, 19-24 de junio de 2006, Universitat de les Illes Balears (pp. 103-116). Palma: AGE y Universitat de les Illes Balears.

Fani, A. (2011). A Condição Espacial. São Paulo: Contexto.

Ferreira, T. \& Soares, B. (2008). Cidade e Planejamento: Uma Ordem em Fragmentos. Espaço \& Geografia, 11(2), 185-202. En http://www.lsie.unb.br/espacoegeografia/index.php/ espacoegeografia/article/view/90

Graham, S. \& Marvin, S. (2001). Splintering Urbanism: Networked Infrastructures, Technological Mobilities and the Urban Condition. Nueva York: Routledge.

Gutiérrez, A. (2008). Los desplazamientos motorizados y el esparcimiento urbano ilimitado. Megapolis, transportes y movilidades. En Megapolis, transportes y movilidades. Confrontaciones (pp. 145-160). Encuentro del 9 de abril de 2008, Universidad de Buenos Aires..

Gutiérrez, A. (2009). Movilidad y acceso: Embarazo y salud pública en la periferia de Buenos Aires.. Ponencia presentada en el xv Congreso Latinoamericano de Transporte Público y Urbano (CLATPU), Buenos Aires. En http://www.filo.uba.ar/contenidos/ investigacion/institutos/geo/ptt/GutierrezClatpu09a.pdf

Haesbaert, R. (2007). O mito da desterritorializção: do "fim dos territórios" á multiterritorialidade (3a ed.). Río de Janeiro: Bertrand Brasil.

Hagerstrand, T. (1991). ¿Qué hay acerca de las personas en la Ciencia Regional? Serie Geográfica, 1, 93-110. En http://bit.ly/1poOeNT

Harvey, D. (1994). La construcción social del espacio y del tiempo: una teoría relacional. Geographical Review of Japan, 67(2), 126-135. En http://geografiacriticaecuador.files. wordpress.com/2013/01/16-harvey.pdf

Harvey, D. (2004). La condición de la posmodernidad: Investigación sobre los orígenes del cambio cultural. Buenos Aires: Amorrotu Editores. 
Hidalgo, R. (2004). De los pequeños condominios a la ciudad vallada: las urbanizaciones cerradas y la nueva geografía social en Santiago de Chile (1990-2000). EURE, 30(91), 29-52. http://dx.doi.org/10.4067/S0250-71612004009100003

Hidalgo, R., Borsdorf, A. \& Sánchez, R. (2007). La expansión residencial amurallada en la reconfiguración metropolitana en Santiago de Chile. Documento presentado en el Taller Nacional sobre "Migración interna y desarrollo en Chile: diagnóstico, perspectivas y políticas", 10 de abril de 2007, Santiago, Chile. Organizado por la Comisión Económica para América Latina y el Caribe (CEPAL), Centro Latinoamericano y Caribeño de Demografía (CELADE), División de Población, con el apoyo y auspicio del Banco Interamericano de Desarrollo (BID).

Hidalgo, R., Bordorf, A., Zunino, H. \& Álvarez, L. (2008). Tipologías de expansión metropolitana en Santiago de Chile: precariópolis estatal y privatópolis inmobiliaria. Scripta Nova. Revista Electrónica de Geografía y Ciencias Sociales, 12 (270).

Hiernaux, D. (2006). ¿Identidades móviles o movilidad sin identidad? En: A. I. Gerais de Lemos, M. L. Silveira \& M. Arroyo (Eds.), Questôes Territoriais Na América Latina, Coleção Edição e Distribuição Cooperativa (pp. 163-175). Buenos Aires: Consejo Latinoamericano de Ciencias Sociales (CLACSO).

Instituto Nacional de Estadísticas (INE), Chile. (2002). xvir Censo Nacional de Población y vi de Vivienda. Santiago de Chile: Autor. En http://www.ine.cl/canales/chile_estadistico/ censos_poblacion_vivienda/censo_pobl_vivi.php

Janoschka, M. (2002). El nuevo modelo de la ciudad latinoamericana: fragmentación y privatización. EURE, 28(85), 11-29. http://dx.doi.org/10.4067/S025071612002008500002

Janoschka, M. (2006). El modelo de ciudad latinoamericana. Privatización y fragmentación del espacio urbano en Buenos Aires: el caso Nordelta. En Welch, M. (Ed.), Buenos Aires a la deriva. Transformaciones urbanas recientes (pp. 96-131). Buenos Aires: Editorial Biblos.

Jarvis, H. (2005). Moving to London time. Household co-ordination and the infrastructure of everyday life. Time \& Society, 14(1): 133-154. doi: 10.1177/0961463X05050302

Jirón, P. (2010a). Repetition and difference: Rhythms and mobile place-making in Santiago de Chile. En T. Edensor (Ed.), Geographies of rhythm: Nature, place, mobilities and bodies (pp. 129-143). Farnham, Surrey Ashgate Publishing Limited.

Jirón, P. (2010b). Mobility practices in Santiago de Chile: The consequences of restricted urban accessibility. En G. Pellegrino (Ed.), The politics of proximity: Mobility and immobility in practice (pp. 133-151). Farnham, Surrey Ashgate Publishing Limited.

Jirón, P. (2010c). On becoming 'la sombra/the shadow'. En M. Büscher, J. Urry, K. Witchger, Mobile Methods (pp. 36-53). Abingdon: Taylor \& Francis Books.

Jirón, P. (2012). Technology and technicians out of control: The implementation of transantiago from a daily mobility point of view. En L. Vannini, C. Budd, P. Fisker, Jiron, P. \& O. Jensen, Technologies of mobility in the Americas. Series Intersections in communications and culture: Global approaches and transdisciplinary perspectives, Vol. 29 (pp. 255277). Nueva York: Peter Lang Publishing Group. 
Jirón, P. \& Cortés, S. (2011). Mobile relations, mobile shadows. Understanding contemporary urban daily living through shadowing techniques. Ponencia presentada en el International Workshop: The Everyday Life of Multi-Local Families. Concepts, Methods and the Example of Post-Separation Families, octubre 20-21 2011, Munich, Alemania.

Jirón, P. \& Iturra, L. (2011). Momentos móviles. Los lugares móviles y la nueva construcción del espacio público. Revista Arquitecturas del Sur, 39, 45-57. En http://vivienda. uchilefau.cl/extension/pdfs/Jiron,Iturra_Momentos_Moviles.pdf

Jirón, P., \& Mansilla, P. (2013). Atravesando la espesura de la ciudad: vida cotidiana y barreras de accesibilidad de los habitantes de la periferia urbana de Santiago de Chile. Revista de Geografia Norte Grande, 56, 53-74. http://dx.doi.org/10.4067/S071834022013000300004

Jirón, P., Lange, C. \& Bertrand, M. (2010). Exclusión y desigualdad espacial: Retrato desde la movilidad cotidiana. Revista del Instituto de la Vivienda, 25(68), 15-57. http://dx.doi. org/10.4067/S0718-83582010000100002

Kaufmann, V. (2002). Re-thinking mobility: Contemporary Sociology. Ashgate: Aldershot, RU.

Kusenbach, M. (2003). Street phenomenology: The go-along as ethnographic research tool. Ethnography, 4(3), 455-485. doi: 10.1177/146613810343007

Lange, C. (2011). Dimensiones culturales de la movilidad urbana. Revista INVI, 26(71), 87106. http://dx.doi.org/10.4067/S0718-83582011000100004

Latham, A. (2004). Researching and writing everyday accounts of the city. An introduction to the diary-photo diary-interview method. En C. Knowles \& P. Sweetman (Eds.), Picturing the social landscape: Visual methods and the sociological imagination (pp. 117131). Londres: Routledge.

Lefebvre, H. (2004). Rhythmanalysis. Space, time and everyday life (Traducido por S. Elden \& G. Moore). Nueva York: Continuum (Texto original: Éléments de rythmanalyse. París: Edicion Sullepse, 1992).

Link, F. (2008). De la policentralidad a la fragmentación en Santiago de Chile. Revista de la Organización Latinoamericana y del Caribe de Centros Históricos, 2, 13-24. En www. redalyc.org/articulo.oa?id=115112535002

Massey, D. (1995). The conceptualization of place. En D. Massey \& P. Jess (Eds.), A place in the world? Places, cultures and globalization (pp. 45-47). Nueva York: Oxford University Press and The Open University.

Massey, D. (2005). For space. Londres: Sage Publications.

Mansilla, P. (2011a). De la racionalidad cartográfica de la escala a las políticas de escala en el proceso de restructuración metropolitano. Revista Espacios, 1, 53-65.

Mansilla, P. (2011b). Autopistas, accesibilidad y desigualdad social: el impacto de las red de autopistas concesionadas sobre la dimensión temporal de las prácticas de movilidad en Santiago de Chile. Revista Geográfica de América Central, 2(47E), 1-12. En http:// www.revistas.una.ac.cr/index.php/geografica/article/view/2096

May, J. \& Thrift, N. (2001). Introduction. En J. May \& N. Thrift (Eds.), Timespace. Geographies of temporality (pp. 1-46). Londres: Routledge.

Naranjo, G. (2009). El rol de la ciudad infiltrada en la reconfiguración de la periferia metropolitana de Santiago de Chile. Estudios Geográficos, 70(266), 205-229. doi:10.3989/estgeogr.0448 
Ortiz, J. \& Améstica, K. (2011). Movilidad espacial y cambios en la sociodemografía en una comuna periférica del Gran Santiago. En S. Escolano \& J. Ortiz, J. (Eds), Sobre la medición de la forma del espacio urbano: Aplicación a Santiago (Chile) y a Zaragoza (España). Zaragoza: Prensas Universitarias de Zaragoza.

Parnreiter, C. (2003). Polarización económica y fragmentación espacial: El caso de México. Actas Latinoamericanas de Varsovia, 26, 173-190.

Prévôt-Schapira, M-F. (2000). Segregación, fragmentación, secesión. Hacia una nueva geografía social en la aglomeración de Buenos Aires. Economía, Sociedad y Territorio, 2(7), 405431. En http://www.redalyc.org/articulo.oa?id=11100702

Prévôt-Schapira, M-F. (2001). Fragmentación social y espacial: conceptos y realidades. Perfiles Latinoamericanos, 19, 33-56. En http://bit.ly/SiLF1G

Prévôt-Schapira, M-F. \& Cattaneo Pineda, R. (2008). Buenos Aires: la fragmentación en los intersticios de una sociedad polarizada. EURE, 34(103), 73-92. http://dx.doi. org/10.4067/S0250-71612008000300004

Rodríguez, A. \& Winchester, L. (2005). Santiago de Chile: una ciudad fragmentada. En C. De Mattos, M. E. Ducci, A. Rodríguez \& G. Yáñez (Eds.), Santiago en la globalización: una nueva ciudad (pp. 115-136). Santiago de Chile: Coedición, SUR Corporación de Estudios Sociales y Educación / Instituto de Estudios Urbanos, P. Universidad Católica de Chile.

Rodríguez, J. (2008). Movilidad cotidiana, desigualdad social y segregación residencial en cuatro metrópolis de América Latina. EURE, 34(103), 49-71. http://dx.doi.org/10.4067/ S0250-71612008000300003

Rodríguez, J. \& Arriagada, C. (2004). Segregación residencial en la ciudad latinoamericana. EURE, 30(89), 5-24. http://dx.doi.org/10.4067/S0250-71612004008900001

Sabatini, F., Cáceres, G. \& Cerda, J. (2001). Segregación residencial en las principales ciudades chilenas: Tendencias de las últimas décadas y posibles cursos de acción. EURE, 27(82), 21-42. http://dx.doi.org/10.4067/S0250-71612001008200002

Sabatini, F., Salcedo, R., Wormald, G. \& Cáceres, G. (2010). Tendencias de la segregación en las principales ciudades chilenas: análisis censal 1982-2002. Santiago de Chile: Instituto Nacional de Estadísticas (INE).

Salvador, J. (2000). Las tensiones espacio-temporales de la vida cotidiana. En: A. Lindón (Coord.), La vida cotidiana y su espacio-temporalidad (pp. 123-146). Barcelona: Anthropos; México: El Colegio Mexiquense, Centro Regional de Investigaciones Multidiscilpinarias, Universidad Nacional Autónoma de México (UNAM).

Santos, M. (1994). Técnica, espaço e tempo. São Paulo: Editora da Universidade do São Paulo (Usp), Coleção Milton Santos.

Secretaría de Transportes (sectra). (2006). Encuesta de Movilidad del Gran Santiago, año 2006. Santiago de Chile: Autor. En http://www.sectra.gob.cl/datos_e_informacion_espacial/ gran_santiago/encuestas_movilidad.html

Sheller, M. \& Urry, J. (2006). The new mobilities paradigm. Environment and Planning A, 38(2), 207-226. En http://www.envplan.com/abstract.cgi?id=a37268

Smith, N. (2001). Nuevo globalismo, nuevo urbanismo. Documents d'anàlisi geogràfica, 38, $15-$ 32. En http://www.raco.cat/index.php/DocumentsAnalisi/article/view/31735

Soja, E. (2000). Postmetropolis. Critical studies of cities and regions. Oxford: Blackwell Publishing. 
Souza, M. Lopez de (2008). Fobópole: O medo generalizado e a militarização da questão urbana. Río de Janeiro: Bertrand Brasil.

Spinney, J. (2007). Cycling the city: movement, meaning and practice. PhD Thesis, Department of Geography, University of London, Londres. Publicada en 2009 en Geography Compass, 7(2), 817-835. doi:10.1111/j.1749-8198.2008.00211.x

Urry J. (2007). Mobilities. Cambridge, uk: Polity Press.

Valdés, E. (2007). Fragmentación y segregación urbana. Aportes teóricos para el análisis de casos en la ciudad de Córdoba. Revista Digital Alfilo, 18. En http://www.ffyh.unc.edu. ar/alfilo/anteriores/alfilo-18/pdf/valdes.pdf

Veiga, D. (2004). Desigualdades sociales y fragmentación urbana. En A. Ribeiro (Ed.), El rostro urbano de América Latina (51-61). Buenos Aires: Consejo Latinoamericano de Ciencias Sociales (CLACso). En http://www.biblioteca.clacso.edu.ar/ar/libros/campus/ poggiese/07veiga.pdf

Veltz, P. (1999). Mundialización, ciudades y territorios. Barcelona: Ariel.

Vidal-Koppman, S. (2009). Fragmentación socioespacial en la periferia de la Región Metropolitana de Buenos Aires. Journal of Latin American Geography, 8(1), 79-97. 